\title{
DIRECT Distances to Local Group Galaxies
}

\author{
Dimitar D. Sasselov \\ Harvard-Smithsonian Center for Astrophysics, \\ 60 Garden St., Cambridge, MA 02138, USA
}

\begin{abstract}
The two nearby galaxies, M31 and M33, are stepping stones for most of our current efforts to understand the evolving universe at large scales. We are undertaking a long term project, called DIRECT, to improve the direct distance estimate to M31 and M33. The massive photometry we have obtained as part of our project over the past 3 years provides us with very good light curves for known and new Cepheid variables, a large number of eclipsing binaries and other variable stars.
\end{abstract}

\section{Distances to Local Group Galaxies}

The distances to nearby Local Group galaxies are not known very well, especially with the new demands of extragalactic stellar astrophysics which are only likely to increase with the advent of several 8-m class telescopes (e.g., Aparicio, Herrero, \& Sanchez 1998). Nearby galaxies are also crucial calibrators to techniques for establishing the extragalactic distance scale and determining the value of $H_{0}$ (Mould et al. 1999). Two important spiral galaxies in our nearest neighborhood are M31 and M33. Yet, their distances are now known to no better than 10-15\%, as there are discrepancies of $0.2-0.3 \mathrm{mag}$ between various distance indicators (e.g. Huterer, Sasselov, \& Schechter 1995; Holland 1998; Stanek \& Garnavich 1998) (see Fig. 1).

Direct distances to M31 and M33 are now achievable, by the use of geometric techniques, with detached eclipsing binaries and Cepheids, and the use of $8-10-\mathrm{m}$ class telescopes for the required spectroscopy. Such direct distances are the ultimate goal of project DIRECT, which we started three years ago (Kaluzny et al. 1998, 1999; Stanek et al. 1998, 1999). The identification of variables suitable for direct study was accomplished by massive photometry; much of it still continues. In this review I describe some results from the massive photometry by project DIRECT in M31 and M33.

\section{Project DIRECT}

The DIRECT project team now consists of K. Z. Stanek (CfA), J. Kaluzny (Warsaw), A. H. Szentgyorgyi (CfA), J. L. Tonry (Hawaii), L. M. Macri (CfA), B. J. Mochejska (Warsaw), and myself. Between September 1996 and November 1999 we have obtained $\sim 170$ nights on the $1.2-\mathrm{m}$ FLWO telescope and 35 nights on the 1.3-m MDM telescope for the project, 23 nights on the 2.1-m KPNO telescope, and four nights on the Keck II 10-m telescope. We have 


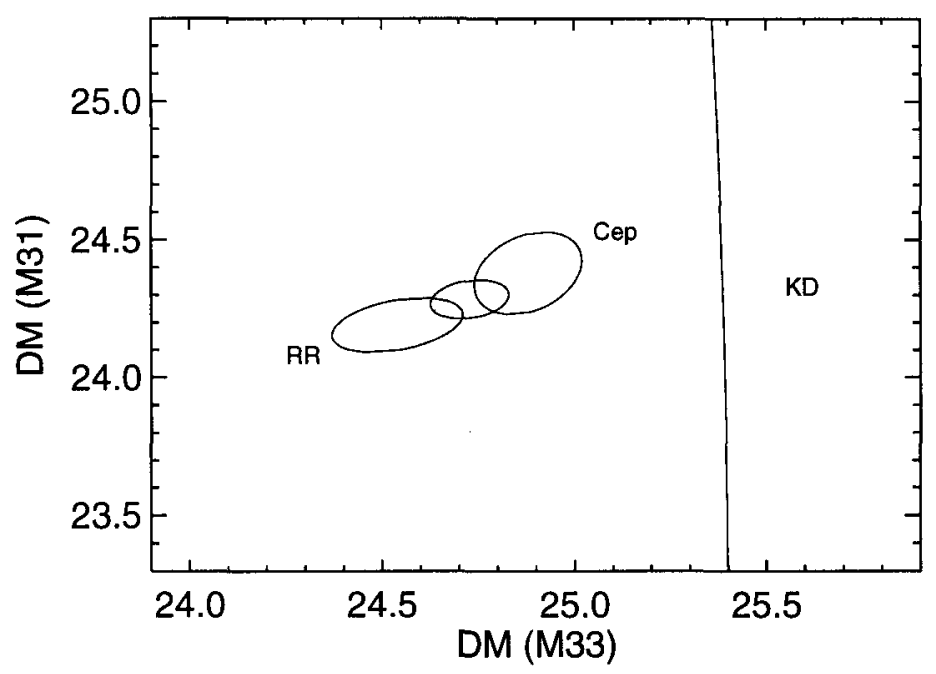

Figure 1. Projections of the distance uncertainty ellipsoids for the nearby galaxies M31 and M33. The RR Lyrae and Cepheid distances are systematically discrepant in both. The unmarked ellipse is the projection of the average distance ellipsoid, based on the simultaneous solution for 15 objects (see Huterer et al. 1995 for details). KD stands for a kinematic distance limit from VLBI of maser sources in M33.

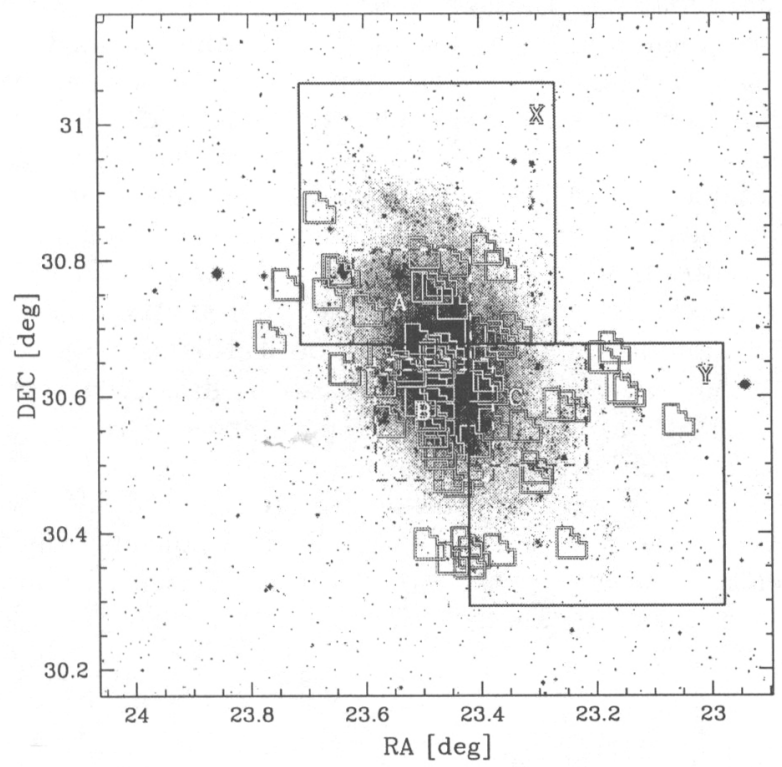

Figure 2. The M33 galaxy with superimposed fields observed by the HST/WFPC2 (small footprints). Also shown are the DIRECT project $11^{\prime} \times 11^{\prime}$ fields ABC monitored for variability in 1996 and 1997 (small rectangles) and $23^{\prime} \times 23^{\prime}$ fields XY monitored in 1998 and 1999. 
completely reduced and analyzed data for five of the fields in M31. We have found in these fields 410 variable stars: 48 eclipsing binaries, 206 Cepheids and 156 other variables; about 350 of these variables are newly discovered. We should stress here that for the first time detached eclipsing binaries were found in M31 by a CCD search. We are completing the reduction of the remaining fields and will continue submitting the next parts of M31/M33 variables catalogs for publication. In Fig. 2 we show the fields observed in M33 by our project in 1996-97 (small rectangles) and 1998-99 (large rectangles).

The moment our papers are submitted for publication, all the variable star light curves and finding charts are made available through the anonymous ftp on cfa-ftp.harvard.edu, in pub/kstanek/DIRECT directory; and also through the $W W W$ at the http://cfa-ww.harvard.edu/ $k$ stanek/DIRECT/.

\section{Results on Cepheids}

So far we have found 206 Cepheids in M31 and 270 in M33. For two of them we have obtained Keck II LRIS spectra suitable for detailed abundance analyses and precise velocities. Our approach has been to obtain very well sampled light curves. This secures the identification of all classes of variable stars, allows meaningful Fourier decompositions for Cepheids to $i=3$, at least, and lets us deal with some issues of metallicity differences and blending in the Cepheid population.

For example, pulsation resonances are known to play an important role in shaping the morphology of the light curves of Cepheids, e.g. the Hertzsprung bump progression centered on period of $10^{d}$, identified with the 2:1 resonance $2 \omega_{0} \approx \omega_{2}$. Not surprisingly, it is very sensitive to the opacity in the Cepheid envelope, a crude sort of asteroseismology, and is a good indicator of the metallicity of a Cepheid sample (Buchler 1997). Thanks to data from the microlensing surveys the metallicity dependence of the resonance center has now been established from Cepheids in the Galaxy, LMC, and SMC (Beaulieu \& Sasselov 1997). The resonance center is shifted by full $2 \mathrm{~d}$ for a $\Delta[\mathrm{Fe} / \mathrm{H}]=0.7$. For such purposes the Fourier technique is entirely model and photometry independent, it only depends on well sampled light curves in an optical band.

The two populations of Cepheids in our M31 data, which we expect to be of different metallicity, are located in two spiral arms. Although our inner arm sample is still small (20), its resonance center is defined and shifted by $\sim 1 \mathrm{~d}$ in the fashion described above. The resonance troughs are filled with outliers in Fields B\&C, due to the sample of high-metallicity Cepheids from the inner arm. The galactocentric distances of the two samples are 4 and $10 \mathrm{kpc}$, respectively. At these distances the abundances in the M31 disk are close to solar and slightly higher.

\section{Correction for Blending}

We are using archival HST images coinciding with our fields (see Fig. 2) to study the environments of the Cepheids discovered by the ground-based observations of project DIRECT. We find that roughly $50 \%$ of our Cepheids are blended with other stars which contribute more than $10 \%$ of the light (in the $V$-band). 


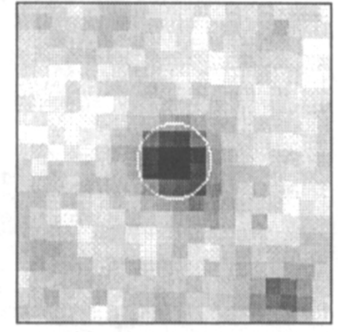

V4125 D31F ground

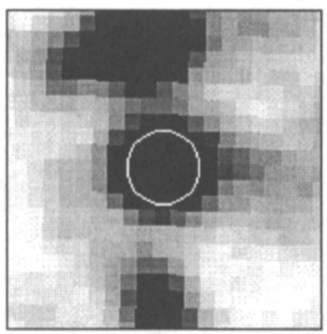

V2203 D31F ground

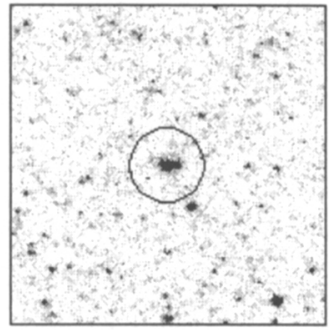

HST

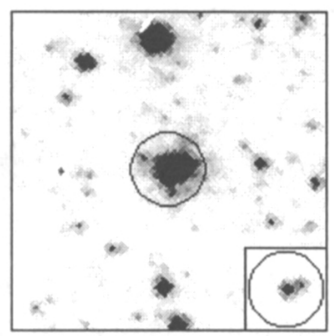

HST

Figure 3. Two examples of blending of Cepheids in M31 found by our current work on DIRECT and archival HST data.

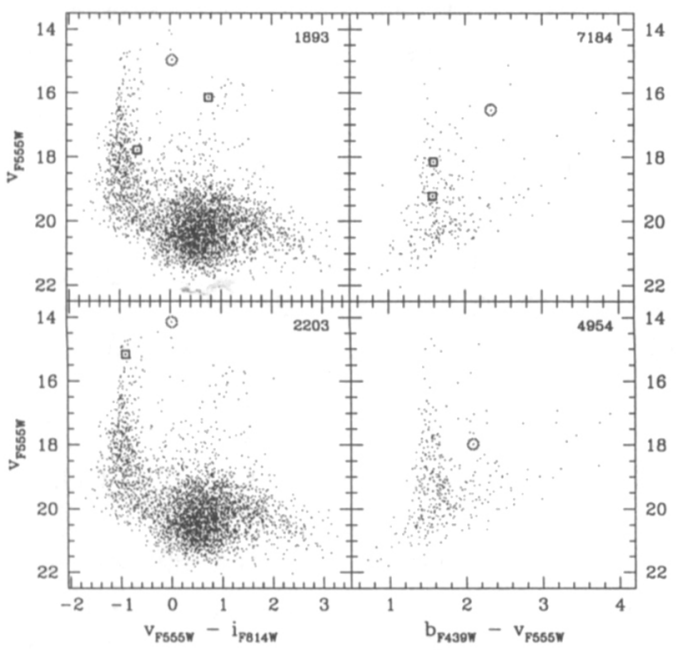

Figure 4. The color-magnitude diagrams for four of the Cepheid blends in M31. In each the Cepheid is marked with a circle. 
Cepheid blending can account for up to $40 \%$ of the measured flux from some of the Cepheids as found in Mochejska et al. (1999), where we report on the sample of 20 Cepheids in M31. The analysis of 90 Cepheids in M33 is practically complete. We find that the average (median) $V$-band flux contribution from luminous companions which are not resolved on the ground-based images is about $19 \%$ (12\%) of the flux of the Cepheids in M31 and 27\% (17\%) in M33. The average (median) $I$-band flux contribution is about $34 \%(24 \%)$ in M33, i.e. there appears to be no bias in the color of the blends.

Our indirect distances to the two galaxies will be easily corrected for this blending. Note that Cepheid V7184 in Fig. 4 was discovered to be a blend by analyzing our ground-based DIRECT light curve, before we had HST data on it (Kaluzny et al. 1998). Cepheid V4954 was flagged accordingly. This shows the usefulness of good light curves, albeit limited to strong blending only.

Our ground-based resolution in M31 and M33 corresponds to the HST resolution at about $10 \mathrm{Mpc}$. Blending leads to systematically low distances to galaxies observed with the HST, and therefore to systematically high estimates of $H_{0}$. We predict the Cepheid blending effects for a galaxy at $\sim 25 \mathrm{Mpc}$ observed by HST to be severe with the corresponding implications to the extragalactic distance scale.

Acknowledgments. This work was supported in part by NSF grant AST-9970812 and NASA grant AR-08354.02-97A from the STScI.

\section{References}

Aparicio, A., Herrero, A., \& Sanchez, F. (ed.) 1998, Stellar Astrophysics for the Local Group, VIII Can. Isl. Winter School (New York: CUP)

Beaulieu, J. P. \& Sasselov, D. 1997, in Variable Stars and the Astrophysical Returns of the Microlensing Surveys, ed. R. Ferlet, J.-P. Maillard, \& B. Raban (Gif-sur-Yvette: Editions Frontières), 193

Buchler, J. R. 1997, in Variable Stars and the Astrophysical Returns of the Microlensing Surveys, ed. R. Ferlet, J.-P. Maillard, \& B. Raban (Gif-sur-Yvette: Editions Frontières), 181

Holland, S. 1998, AJ, 115, 1916

Huterer, D., Sasselov, D., \& Schechter, P. 1995, AJ, 100, 2705

Kaluzny, J., Stanek, K., Krockenberger, M., Sasselov, D., et al. 1998, AJ, 115, 1016

Kaluzny, J., Mochejska, B., Stanek, K., et al. 1999, AJ, 118, 346

Mochejska, B., Macri, L., Sasselov, D., \& Stanek, K. 1999, AJ, submitted [astro$\mathrm{ph} / 9908293]$

Mould, J., Huchra, J., Freedman, W., et al. 1999, preprint [astro-ph/9909260]

Stanek, K. \& Garnavich, P. 1998, ApJ, 503, L131

Stanek, K., Kaluzny, J., Krockenberger, M., et al. 1998, AJ, 115, 1894

Stanek, K., Kaluzny, J., Krockenberger, M., Sasselov, D., et al. 1999, AJ, 117, 2810 